AUTHOR QUERY FORM

\begin{tabular}{|l|l|l|}
\hline & Please e-mail your responses and anycorrections to: \\
& & \\
& & \\
\hline ELSEVIER & Article Number: 22588 & E-mail: correctionsaptara @ elsevier.com \\
\hline
\end{tabular}

Dear Author,

Please check your proof carefully and mark all corrections at the appropriate place in the proof (e.g., by using on-screen annotation in the PDF file) or compile them in a separate list. Note: if you opt to annotate the file with software other than Adobe Reader then please also highlight the appropriate place in the PDF file. To ensure fast publication of your paper please return your corrections within 48 hours.

Your article is registered as a regular item and is being processed for inclusion in a regular issue of the journal. If this is NOT correct and your article belongs to a Special Issue/Collection please contact p.kumar.7@elsevier.com immediately prior to returning your corrections.

For correction or revision of any artwork, please consult http://www.elsevier.com/artworkinstructions

Any queries or remarks that have arisen during the processing of your manuscript are listed below and highlighted by flags in the proof. Click on the ' $Q$ ' link to go to the location in the proof.

\begin{tabular}{|c|c|c|}
\hline $\begin{array}{l}\text { Location } \\
\text { in article }\end{array}$ & \multicolumn{2}{|c|}{$\begin{array}{l}\text { Query / Remark: click on the Q link to go } \\
\text { Please insert your reply or correction at the corresponding line in the proof }\end{array}$} \\
\hline Q1 & \multicolumn{2}{|c|}{$\begin{array}{l}\text { AU: The author names have been tagged as given names and surnames (surnames are highlighted } \\
\text { in teal color). Please confirm if they have been identified correctly. }\end{array}$} \\
\hline Q2 & \multicolumn{2}{|c|}{ AU: Please check the sentence "We have also found that.." and correct if necessary. } \\
\hline Q3 & \multicolumn{2}{|c|}{$\begin{array}{l}\text { AU: Figs. [3-8] have been submitted as color images; however, the captions have been reworded } \\
\text { to ensure that they are meaningful when your article is reproduced both in color and in black and } \\
\text { white. Please check and correct if necessary. }\end{array}$} \\
\hline \multirow[t]{2}{*}{ Q4 } & \multicolumn{2}{|c|}{$\begin{array}{l}\text { AU: This section comprises references that occur in the reference list but not in the body of the } \\
\text { text. Please cite each reference in the text or, alternatively, delete it. Any reference not dealt with } \\
\text { will be retained in this section. }\end{array}$} \\
\hline & $\begin{array}{l}\text { Please check this box or indicate your approval if } \\
\text { you have no corrections to make to the PDF file }\end{array}$ & \\
\hline
\end{tabular}

Thank you for your assistance. 


\section{Windows for escaping particles in quartic galactic potentials}

\section{罯 F. Navarr $\equiv$ \\ Department of Applied Mathematics, University of Alicante, Carretera San Vicente del Raspeig, s/n, 03690 San Vicente, Alicante, Spain}

\section{A R T I C L E I N F O}

\section{Keywords:}

Galactic potentials

Periodic orbits

Escapes

Hamiltonian systems

\begin{abstract}
A B S T R A C T
We investigate the shape of the windows through which stars may escape from a galaxy modeled by a bi-symmetrical potential made up of a two-dimensional harmonic oscillator with quartic perturbing terms. The escape from the potential well is governed by the unstable periodic orbits in the openings of the potential. The unstable and stable manifolds to these periodic orbits reveal the way test particles escape from the potential well. Our main objective is to compute accurately these manifolds to analyze the shapes and sizes of the windows of escape, founding that they consist of a "main windowa" and of a hierarchy of secondary spiral windows. We have also found that the shape and the way this hierarchy is constructed depend on the energy of the system. This study is performed through the study $y_{\mathcal{L}}$ of intersections of stable and unstable manifolds in the $x-\dot{x}$ Poincaré phase plane.
\end{abstract}

(C) 2017 Published by Elsevier Inc.

\section{Introduction}

The phenomenon of escapes from a dynamical system, especially the escape of stars from stellar systems has been an active field of research during the last decades [1-8,11,12,17-19,21,22]. In [11], Contopoulos explored escapes from dynamical systems representing the central part of perturbed galaxies, with Hamiltonian [20]

$$
\mathcal{H}=\frac{1}{2}\left(\dot{x}^{2}+\dot{y}^{2}\right)+\frac{1}{2}\left(x^{2}+y^{2}\right)-\mu x^{2} y^{2} .
$$

This system have quadruple symmetry and four openings to infinity. Every opening is bridged by an unstable periodic orbit called a Lyapunov orbit, which governs the escape to infinity from the potential well. The asymptotic curves of the Lyapunov periodic orbits make infinite rotations around some "limiting curves". The proportion of escaping orbits and the direction of escape depend on the topology of the asymptotic surfaces of the Lyapunov periodic orbits. This is investigated in [12] by Contopoulos and Kauffman. The "basins" of escape toward different directions, and of the fast and the slow escapes for various values of the perturbation parameter $(\mu)$ are determined. In 1996, Siopis et al. [19] performed a numerical study of the escape properties of three two-dimensional, time-independent potentials possessing different symmetries. It was found, for all three cases, that (i) there is a rather abrupt transition in the behaviour of the late-time probability of escape, when the value of a coupling parameter, $\mu$, exceeds a critical value, $\mu_{2}$. For $\mu>\mu_{2}$, it was found that (ii) the escape probability manifests an initial convergence towards a nearly time-independent value, $p_{0}(\mu)$, which exhibits a simple scaling that may be universal. However, (iii) at later times the escape probability slowly decays to zero as a power-law function of time. Finally, it was found that (iv) in a statistical sense, orbits that escape from the system at late times tend to have short time Lyapunov exponents which are lower than for orbits that escape at early times. Navarro and Henrard [18] examined the

E-mail address: jf.navarro@ua.es 
shape of the windows through which test particles may escape in the simplified Hamiltonian system (1). Here, the authors analyzed the shapes and sizes of the windows of escape of stars from a simplified galactic model, founding that they consist of a "main window" and of a hierarchy of secondary windows. A very large part of the main window is actually made of "just passing through"stars and may not be very interesting for galactic studies. Hence the importance of the secondary windows, their intricate spiral structures and the fractality of the basin boundaries.

In 2004, Contopoulos and Efstathiou [13] studied in detail the form of the asymptotic manifolds of a central unstable periodic orbit. The form of the escape regions and the infinite spirals of the asymptotic manifolds around the escape regions were given, as well as the computation of the escape rate for different values of the energy and the percentage of orbits that escape after a finite number of iterations. The problem of the escape in galactic potentials has been recently revisited by Zotos [21,22], who has investigated the structure of the phase space of the dynamical system described by (1).

In [5], Barrio and coworkers explored the appearance of different kinds of fractal structures in Hénon-Heiles potentials in the unbounded range. In [6], they found that open Hamiltonians present safe bounded regular regions inside the escape region that have significant size and that can be located with precision. Therefore, it is possible to find regions of nonzero measure with stable periodic or quasi-periodic orbits far from the last KAM tori and far from the escape energy. Later, in [7], the authors make use of recent computational techniques in the numerical study of qualitative properties of Hamiltonian systems of two degrees of freedom. These numerical methods are based on the computation of the OFLI2 Chaos Indicator, the Crash Test and exit basins and the skeleton of symmetric periodic orbits.

The aim of this article is to better understand the properties of the escape of orbits in a simple local galactic Hamiltonian describing the motion near the center of a elliptical galaxy. In the present work, we study the potential

$$
W(x, y)=\frac{1}{2}\left(\omega_{1}^{2} x^{2}+\omega_{2}^{2} y^{2}\right)-\mu\left[\beta\left(x^{4}+y^{4}\right)+2 \alpha x^{2} y^{2}\right],
$$

where $\omega_{1}, \omega_{2}$ are the unperturbed frequencies of oscillation along the $x$ and $y$ axis respectively, $\mu>0$ is the perturbation strength, and $\alpha$ and $\beta$ are positive parameters [9]. Here, we analyze the case $\omega_{1}, \omega_{2}=\omega=1$, that is, the $1: 1$ resonance case. A description of the Hamiltonian to the potential (2), given by

$$
\mathcal{H}=\frac{1}{2}\left(\dot{x}^{2}+\dot{y}^{2}\right)+\frac{1}{2}\left(x^{2}+y^{2}\right)-\mu\left[\beta\left(x^{4}+y^{4}\right)+2 \alpha x^{2} y^{2}\right],
$$

can be found in [10]. It is actually a member of the Verhulst family of galactic potentials (see [20] for a review).

We will compute the asymptotic manifolds to the Lyapunov periodic orbits in the openings of the potential in order to show that the mechanism described in [18] is valid also in this case. As in the simplified model described there, we show that the intricate spiral structures of the secondary windows governs the rate of escape: there are "first ordex", infinitely winding spirals, but also "second ordea" spirals composed themselves of an infinity of layers, "third orden" spirals formed by an infinity of second order spirals, and so on.

We have alse found that the shape and size of the spirals, as well as the number of "main" first order spirals, depend on the energy of the system. We show this fact carrying out the analysis for two values of the energy.

In order to investigate the size, shape and properties of the regions of phase space leading to escape, it is necessary to understand the geometry of the stable manifolds to the "guardian" periodic orbits. We will do so by investigating the intersections of the stable and unstable manifolds with a surface of section. This has been the strategy of Contopoulos and coworkers [11,12] and Navarro and Henrard [18].

In our analysis, we will take as surface of section the plane $y=0$, and use a sixth order expansion of the stable and unstable manifolds in order to obtain precise initial conditions far enough from the unstable "guardian orbit" to start a safe computation of these manifolds.

\section{Symmetries of the problem}

There are two different cases of the $x y$ plane in the Hamiltonian (3), depending on the relation between $\alpha$ and $\beta$ : (a) $\alpha>\beta$. (b) $\beta>\alpha$. In all these situations, there is a critical value of the energy $\left(h_{c}\right)$ such that, for larger values of $h$, the potential well opens up to infinity and test particles may escape. This critical value is given by

$$
h_{c}=\frac{1}{8 \mu(\alpha+\beta)}
$$

when $\alpha>\beta$, and

$$
h_{c}=\frac{1}{16 \mu \beta}
$$

if $\alpha<\beta$. These values can be computed using the method described in [9]. The curves of zero velocity of the Hamiltonian system (3) are given by the relation

$$
f\left(x^{2}, y^{2}\right)=h-\frac{1}{2}\left(x^{2}+y^{2}\right)+\mu\left[\beta\left(x^{4}+y^{4}\right)+2 \alpha x^{2} y^{2}\right]=0 .
$$


The points of the $x y$ plane where the curve of zero velocity opens are the saddle-points of (4). In order to calculate these points, we first solve the system

$$
\begin{aligned}
& \frac{\partial f}{\partial x}=4 \mu \alpha x y^{2}-x-4 \mu \beta x^{3}=0 \\
& \frac{\partial f}{\partial y}=4 \mu \beta y^{3}-y+4 \mu \alpha x^{2} y=0 .
\end{aligned}
$$

The solutions of (5) are the critical points of (4). The saddle-points of (4) are those of the critical points satisfying the condition

$$
S=\left(\frac{\partial^{2} f}{\partial x^{2}}\right)\left(\frac{\partial^{2} f}{\partial y^{2}}\right)-\left(\frac{\partial^{2} f}{\partial x \partial y}\right)^{2}<0 .
$$

In addition to the trivial solution $x=y=0$, there are three sets of solutions to (5). If $\alpha>\beta$, there are four saddle-points in the $x y$ plane, given by

$$
x^{2}=\frac{\alpha-\beta}{4 \mu\left(\alpha^{2}-\beta^{2}\right)}, \quad y^{2}=\frac{\alpha-\beta}{4 \mu\left(\alpha^{2}-\beta^{2}\right)} .
$$

Substituting the solution (6) into Eq. (4), we get the critical value

$$
h_{c}=\frac{1}{8 \mu(\alpha+\beta)} \text {. }
$$

69 If $\alpha<\beta$, we get two sets of two saddle-points, given by

$$
x=0, \quad y^{2}=\frac{1}{4 \mu \beta},
$$

and

$$
x^{2}=\frac{1}{4 \mu \beta}, \quad y=0 .
$$

If we substitute Eq. (7) (or (8)) into Eq. (4), we get the critical value

$$
h_{c}=\frac{1}{16 \mu \beta} \text {. }
$$

In our analysis, we will consider the following values of the parameters of the system: $\mu=2.64, \alpha=1.2$ and $\beta=0.8$. The critical value of the energy associated to these values of the parameters is $h_{c}=0.0236742$. For each larger value of $h$, there is an unstable periodic orbit across the opening, bouncing back and forth between the two "walls" of the pass (see Fig. 1).

It has been long recognized $[11,18]$ that these unstable periodic orbits are, in some sense, the guardians of the pass: orbits going through the pass aresshepherded by the stable and unstable manifolds of the periodic orbit. This can be explained in the following way: a continuous variation of initial conditions may lead from an orbit which "bounces back" in the pass and returns inside the well to an orbit which passes through, a very non-continuous behavior. The only way this can happen is that somewhere between these two orbits there is a third one which stays in the pass for an infinite amount of time. Indeed, the general solution $X(x(0), t)$ is a continuous function of the initial condition $x(0)$ for any finite time $t$.

Due to the symmetries of the potential, the well opens up, for values of the energy larger than the critical value $h_{c}$, at four places along the diagonals of the configuration space $(x= \pm y)$ (left panel of Fig. 1). The phase space of Hamiltonian (3) is symmetric with respect to each of the two coordinates $(x, y)$ (and their velocities) and with respect to time (and velocities) reversal, i.e. if $(x(t), y(t), \dot{x}(t), \dot{y}(t))$ is an orbit, then the following are also orbits

$$
\begin{array}{rrrrr}
\text { (a) } & x(t), & y(t), & \dot{x}(t) & , \dot{y}(t), \\
\text { (b) } & -x(t), & y(t), & -\dot{x}(t) & \dot{y}(t), \\
\text { (c) } & x(t), & -y(t), & \dot{x}(t), & -\dot{y}(t), \\
\text { (d) } & -x(t), & -y(t), & -\dot{x}(t), & -\dot{y}(t), \\
\text { (e) } & x(-t), & y(-t), & -\dot{x}(-t), & -\dot{y}(-t), \\
\text { (f) } & -x(-t), & y(-t), & \dot{x}(-t), & -\dot{y}(-t), \\
\text { (g) } & x(-t), & -y(-t), & -\dot{x}(-t), & \dot{y}(-t), \\
\text { (h) } & -x(-t), & -y(-t), & \dot{x}(-t), & \dot{y}(-t) .
\end{array}
$$

Hence it is enough to compute the sections of the stable manifold to one periodic orbit (let us take as (a) the stable manifold to the periodic orbit in the upper-right corner) in order to know by symmetry the sections of the stable and unstable manifolds of the four guardian periodic orbits. This is shown in Fig. 2. 

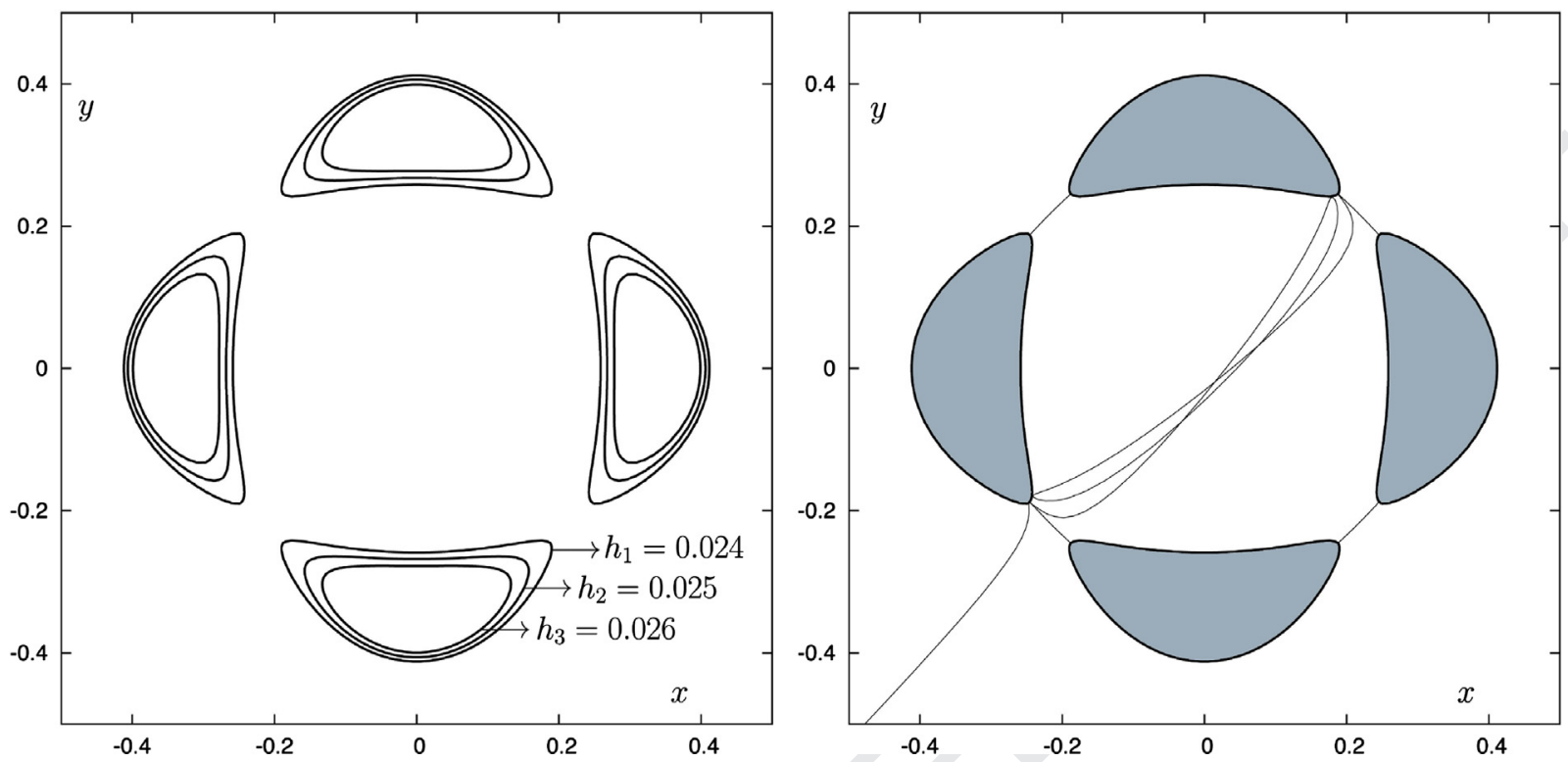

Fig. 1. Curves of zero velocity for $\mu=2.64, \beta=0.8, \alpha=1.2$ and different values of the energy larger than the critical value: $h_{1}=0.024, h_{2}=0.025$ and $h_{3}=0.026$ (left panel). As the energy of the system grows, the windows of the potential well become wider. In the right panel, we show an orbit belonging to the stable manifold to the periodic orbit "guarding" the upper-right opening in the potential well for $\mu=2.64, \beta=0.8, \alpha=1.2$ and $h=0.024$. The guardian orbit is the almost straight line barring the opening. The orbit belonging to the stable manifold is computed backward in time starting from initial conditions computed from a sixth order approximation

Table 1

Initial conditions of the periodic orbits.

\begin{tabular}{llll}
\hline$x_{0}=y_{0}$ & $\dot{x}_{0}=-\dot{y}_{0}$ & $T / 2$ & $h$ \\
\hline 0.21734562531516654 & -0.01805225411091489 & 5.02737847236132162 & 0.024 \\
0.21654774943831516 & -0.03644103615670809 & 5.63431613577672052 & 0.0249 \\
\hline
\end{tabular}

\section{Computation of thestable and unstable manifolds}

Due to the instability of the periodic orbit, we have determined one of them for a value of the energysslightly larger than the critical value following a geometrical approach, and then we have continued the family of periodic orbits taking the energy as parameter. In our study, we analyze the periodic orbits given by the initial conditions we show in Table 1 .

As mentioned in the introduction, the stable manifolds to the "guardian" unstable periodic orbits form the boundaries of the escape windows we wish to analyze. For the computation of the initial part of these stable manifolds (and of the unstable manifolds as well), we shall follow the scheme proposed by Deprit and Henrard [15]. The stable manifold to a periodic orbit $\left(x^{*}(t), y^{*}(t)\right)$ of period $T$ is represented by series

$$
\begin{aligned}
& x(t, \epsilon)=x^{*}(t)+\epsilon u(t), \quad u(t)=\sum_{j \geq 1} \epsilon^{j-1} e^{-j a t} x_{j}(t), \\
& y(t, \epsilon)=y^{*}(t)+\epsilon v(t), \quad v(t)=\sum_{j \geq 1} \epsilon^{j-1} e^{-j a t} y_{j}(t),
\end{aligned}
$$

where the coefficients $x_{j}(t)$ and $y_{j}(t)$ are periodic with period $T$ and $a$ is the (positive) characteristic exponent of the periodic orbit. The unstable manifold is represented by similar series with the exponent $-a$ replaced by $+a$. When $\epsilon$ is equal to zero, we recover the periodic orbit. Substituting the series (10) ip the differential equations derived from the Hamiltonian (3), we obtain

$$
\begin{aligned}
& \ddot{u}=W_{x x}^{*} u+W_{x y}^{*} v+\sum_{i \geq 1} \epsilon^{i-1} \sum_{j \geq 0} c_{i}^{j}\left(\frac{\partial^{i} W}{\partial x^{j} \partial y^{i-j}}\right)^{*} u^{j} v^{i}, \\
& \ddot{v}=W_{y x}^{*} u+W_{y y}^{*} v+\sum_{i \geq 1} \epsilon^{i-1} \sum_{j \geq 0} c_{i}^{j}\left(\frac{\partial^{i+j+1} W}{\partial x^{j} \partial y^{i-j}}\right)^{*} u^{j} v^{i},
\end{aligned}
$$

where we have used the notation $f^{*}$ for the function $f$ evaluated along the periodic solution $\left[f^{*}(t)=f\left(x^{*}(t), y^{*}(t)\right)\right]$. Expanding $u(t)$ and $v(t)$ in powers of $\epsilon$, the Eq. (11) lead to a recursive set of linear equations for the coefficients $x_{k}(t)$ 

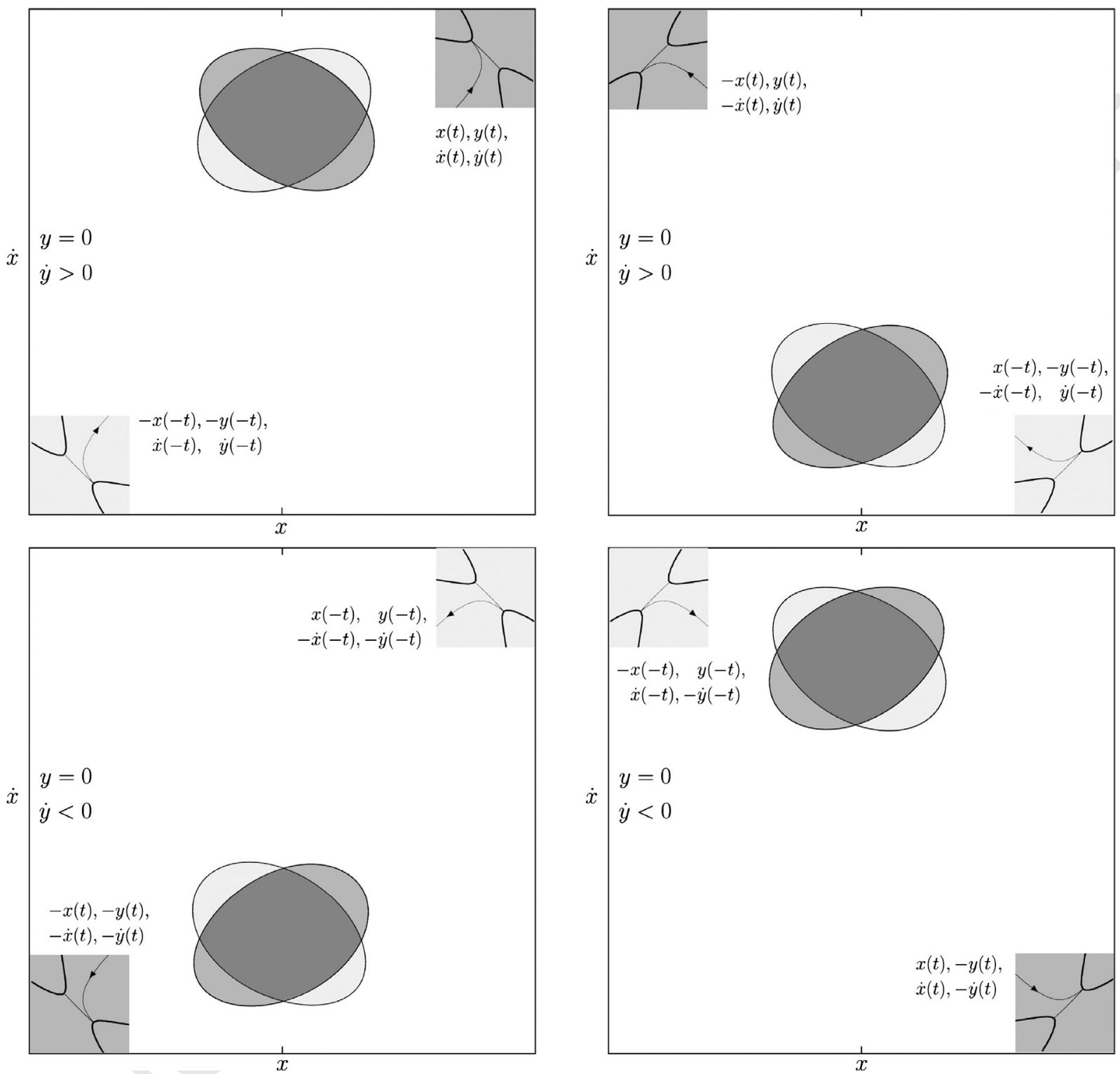

Fig. 2. The first intersection of the two surfaces of section $(y=0, \dot{y}>0)$ and $(y=0, \dot{y}<0)$ with the symmetrics (enumerated in Eq. (9)) of the (internal part of the) stable manifold. The areas in medium grey correspond to orbits leaving the potential well. Areas in light grey correspond to orbits coming from "outside" and which, as a consequence, do not have any antecedents on the surfaces of section. Of course areas with dark grey correspond to orbits "just passing through" the potential well.

and $y_{k}(t)$,

$$
\begin{aligned}
& k^{2} a^{2} x_{k}+2 k a \dot{x}_{k}+\ddot{x}_{k}=W_{x x}^{*} x_{k}+W_{x y}^{*} y_{k}+\Phi_{k}(t), \\
& k^{2} a^{2} y_{k}+2 k a \dot{y}_{k}+\ddot{y}_{k}=W_{x y}^{*} x_{k}+W_{y y}^{*} y_{k}+\Psi_{k}(t),
\end{aligned}
$$

where the periodic functions $\Phi_{k}(t)$ and $\Psi_{k}(t)$ are the contributions of the summations over $j$ in (6) multiplied by $e^{j a t}$. These contributions can readily be computed at order $k$ and are periodic if the $x_{\ell}(t)$ and $y_{\ell}(t)$ (for $\ell<k$ ) are known and periodic.

For $k=1$ the functions $\Phi_{k}(t)$ and $\Psi_{k}(t)$ vanishes and the system (12) is singular. A non-trivial solution is given by the solution of the linear system of variational equations corresponding to the eigenvalue $a$ of the principal matrix. In order to compute it we integrate numerically the two isoenergetic solutions of the variational equations, the initial conditions of which are perpendicular to the initial velocity of the periodic orbit (see [15]). In this way we avoid the eigenspace corresponding to the double zero-eigenvalue. 
The initial conditions are

$$
u(0)=v(0)=0, \quad \dot{u}(0)=-\dot{y}^{*}(0), \quad \dot{v}(0)=\dot{x}^{*}(0),
$$

and

$$
u(0)=-\dot{y}^{*}(0), \quad v(0)=\dot{x}^{*}(0), \quad \dot{u}(0)=\sigma \dot{x}^{*}(0), \quad \dot{v}(0)=\sigma \dot{y}^{*}(0),
$$

with

$$
\sigma=\frac{1}{V^{3}}\left(\dot{x}^{*}(0) W_{y}^{*}(0)-\dot{y}^{*}(0) W_{x}^{*}(0)\right),
$$

where $V$ is the initial velocity on the periodic orbit. We select as solution the linear combination of these two variations which, after a period $T$, reproduces its initial condition multiplied by the factor $\exp (a T)$.
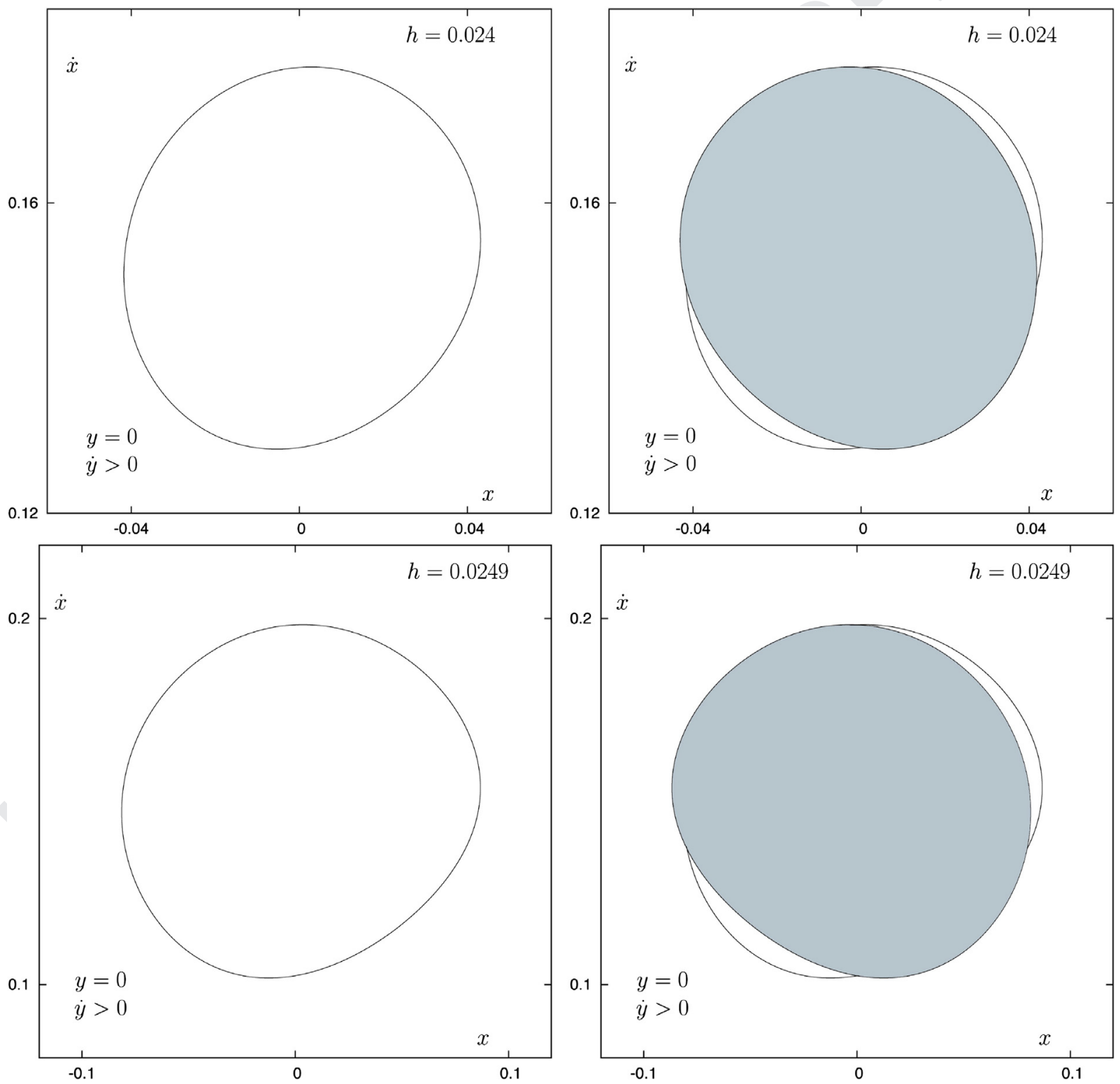

Fig. 3. First section $(y=0, \dot{y}>0)$ of the stable manifold to the first quadrant guardian periodic orbit (on the left panel), for $h=0.024$ (first row) and $h=0.0249$ (second row). On the right panel we have also reproduced (in light blue) the section of the unstable manifold to the third quadrant periodic orbit. The area inside both sections corresponds to orbits coming from infinity from lower-left, passing through the potential well once, and disappearing to infinity to the upper-right. The two remaining crescents (in white) will have antecedents on the surface of section (Fig. 4). (For interpretation of the references to color in this figure legend, the reader is referred to the web version of this article.) 
The periodic orbit and this solution of the variational equations are expanded in Fourier series and thereby the recursive Eq. (12) can be written as a recursive set of linear systems for the Fourier coefficients of the periodic functions $x_{j}(t)$ and $y_{j}(t)$.

\section{The boundaries of the eescapewindows}

We have integrated numerically and backward (using Lie series of order 20 [16]) some 1000000 orbits belonging to the stable manifold to the first quadrant guardian, until they cross the hyperplane $y=0$ (with $\dot{y}>0$ ). The initial conditions were computed by keeping the value of $\epsilon \exp (a t)$ fixed at 0.0001 and choosing 1000000 equidistant values of the initial time $t$.

In Fig. 3 (left panel), we show the first section of the stable manifold to the first quadrant guardian periodic orbit for two different values of the energy, $h=0.024$ and $h=0.0249$. The resulting section is very similar in both cases. By symmetry we can also plot the trace of the unstable manifold to the third quadrant guardian (right panel). We observe that the two "rings" intersect. Orbits starting inside both rings are coming from the lower-left infinity and are going to the upper-right infinity.
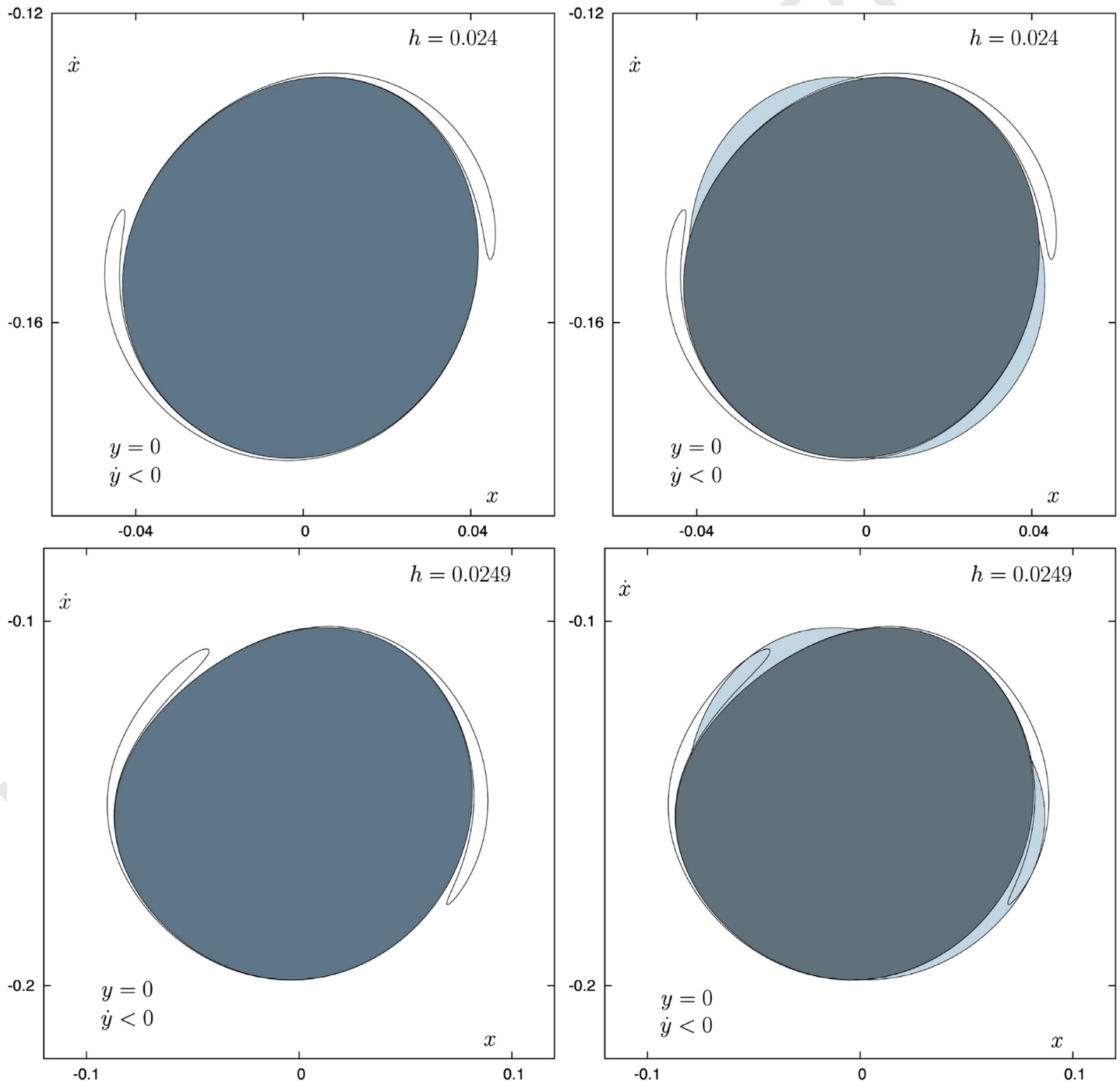

Fig. 4. Second section $(y=0, \dot{y}<0)$ of the stable manifold to the upper-right guardian periodic orbit (left panel), for $h=0.024$ (first row) and $h=0.0249$ (second row). The section is composed of two tongues, images of the two crescents of the right panel of Fig. 3, winding around the stable manifold to the third quadrant periodic orbit (shown in dark blue). Also shown (in light blue) is the section of the unstable manifold to the upper-right guardian periodic orbit. (For interpretation of the references to color in this figure legend, the reader is referred to the web version of this article.) 


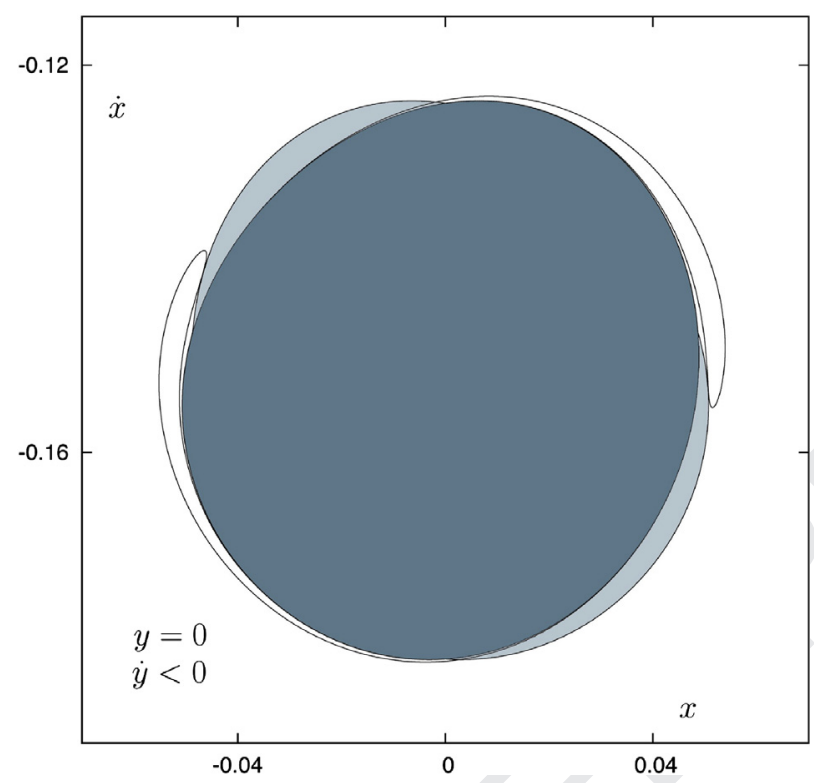

Fig. 5. Second section $(y=0, \dot{y}<0)$ of the stable manifold to the upper-right guardian periodic orbit, for $h^{*}=0.0241200017$. The section is composed of two tongues, winding around the stable manifold to the third quadrant periodic orbit (shown in dark blue). Also shown (in light blue) is the section of the unstable manifold to the upper-right guardian periodic orbit, tangent to the tongues of the stable manifold. (For interpretation of the references to color in this figure legend, the reader is referred to the web version of this article.)

They just pass through the center of the galaxy. On the other hand, orbits starting from one of the two crescents shown in the right panel of Fig. 3 originate inside the galaxy (and of course escape in the future by the upper-right window), and we can consider their previous intersections with the surface of section.

The second intersection, shown in Fig. 4 (left panel), is composed of two "tongues" which spiral around the stable manifold to the third quadrant guardian orbit. The spirals are infinite, but of course we have computed (and shown in Fig. 4) only a part of them. We also show in Fig. 4 the ring created by the unstable manifold to the first quadrant orbit (Fig. 2). Orbits starting inside this ring, and inside one of the two tongues, come from infinity by the upper-right window and leave the galaxy by the same window after two crossings of the axis $y=0$. Orbits inside the tongues but outside the light blue area of Fig. 4 (right panel) have a previous intersection with the surface of section.

Let us remark that there is a difference in the way the intersection between the stable manifold to the upper-right guardian periodic orbit and the unstable manifold to the upper-right guardian periodic orbit takes place for $h=0.024$ and $h=0.0249$. For $h=0.024$, the big lobe of the tongue of the stable manifold does not intersect the unstable manifold (Fig. 4, right panel, first row). However, this part of the tongue of the stable manifold is completely covered by the unstable manifold to the upper-right guardian periodic orbit for $h=0.0249$, as it is shown in Fig. 4 (right panel, second row). In both cases, as commented before, orbits inside the tongues but outside the light blue area of Fig. 4 (right panel) have a previous intersection with the surface of section. There exists a value of $h^{*} \in(0.024,0.0249)$ such that the lobe of the second section of the stable manifold to the upper-right guardian periodic orbit results to be tangent to the unstable manifold to the upper-right periodic orbit. This bifurcation value is $h^{*}=0.0241200017$. In Fig. 5, we show the second section for this value of the energy.

Following these later orbits backward, we compute the third section (see Fig. 6). As a consequence of the way in which the second section of the stable manifold to the upper-right guardian periodic orbit intersects the unstable manifold to the upper-right periodic orbit, for the two values of the energy, the shape of the third section is completely different for both values.

Let us analyze first the third section for $h=0.024$. In this case, the section is composed of two "simple" tongues (the "hook-shaped" ones) plus two "complex" tongues. The complex ones are "Russian dolls", composed of "subtongues" embedded inside each other. Only a few of these subtongues have been computed, but there are an infinity of those subtongues. All these tongues are infinitely winding around the stable manifold to the first quadrant guardian (already shown in Fig. 3). Orbits starting inside this later manifold (in the area shown in dark blue grey) are those which escape directly by the first quadrant window.

In Fig. 7, we show a detail of the composition of the "Russian dolls" structure. The fate of the orbits starting inside the complex tongues alternates according to the parity of the number of subtongues in which they are embedded. Those starting from the area shown in black and grey tones in Fig. 7 (right panel) are mapped on the pieces of the tongues of the second section which are not inside the light blue area (Fig. 4, right panel, first row). 

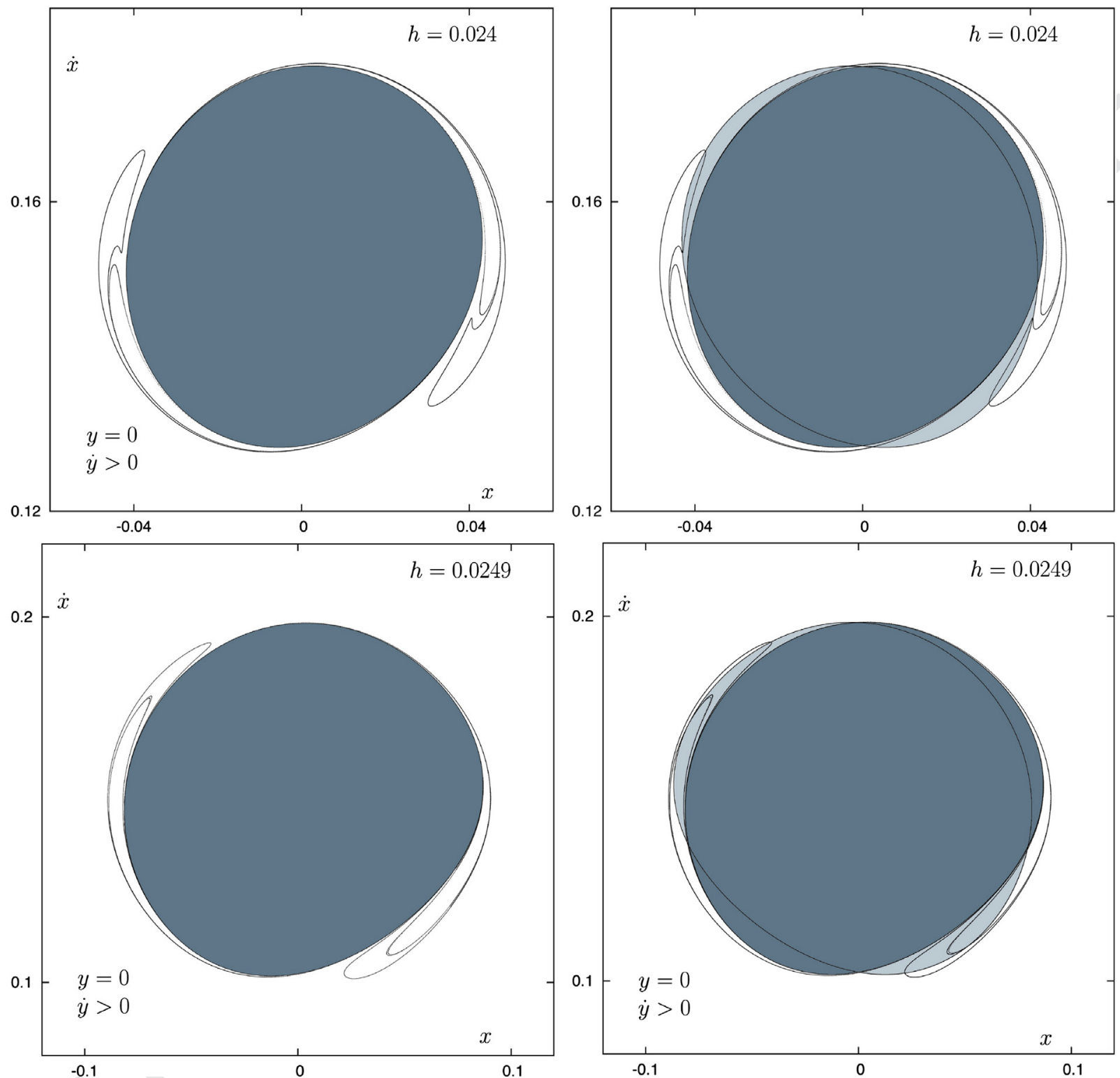

Fig. 6. Third section $(y=0, \dot{y}>0)$ of the stable manifold to the first quadrant periodic orbit, for $h=0.024$ (first row) and $h=0.0249$ (second row). The section for $h=0.024$ is composed of two simple tongues and two complex ones. The simple tongues are the harpoon-shaped ones. The complex tongues are "Russian dolls" composed of an infinity of "subtongues" embedded inside each other. All these tongues wind around the stable manifold to the first quadrant (in dark blue in left panel). In the right panel, we also represent in light blue the unstable manifold to the third quadrant periodic orbit. The section for $h=0.0249$ has a simpler structure. There are two complex tongues, "Russian dolls" composed of an infinity of "subtongues" embedded inside each other. (For interpretation of the references to color in this figure legend, the reader is referred to the web version of this article.)

When $h=0.0249$, the section is composed of two "complex" tongues. These tongues, as before, are "Russian dolls", composed of subtongues' embedded inside each other. We have only computed a few of these subtongues, but there are an infinity of them. All these tongues are infinitely winding around the stable manifold to the first quadrant guardian periodic orbit (already shown in Fig. 3). Orbits starting inside this later manifold (in the area shown in dark blue grey) are those which escape directly by the first quadrant window.

In Fig. 8, we represent a detail of the third section, to analyze the fate of the orbits starting inside the complex tongues. The orbits starting from the area shown in grey tones in Fig. 8 (right panel) are mapped on the pieces of the tongues of the second section which are not inside the light blue area (Fig. 4, right panel, second row).

We also reproduce in Fig. 6 the trace of the unstable manifold to the third quadrant periodic orbit (shown in light blue grey in the right panel of Fig. 3). Orbits inside it and inside the tongues come from infinity by the lower-left window; the others have antecedents on the surface of section and we could compute them backward to a fourth intersection with the 

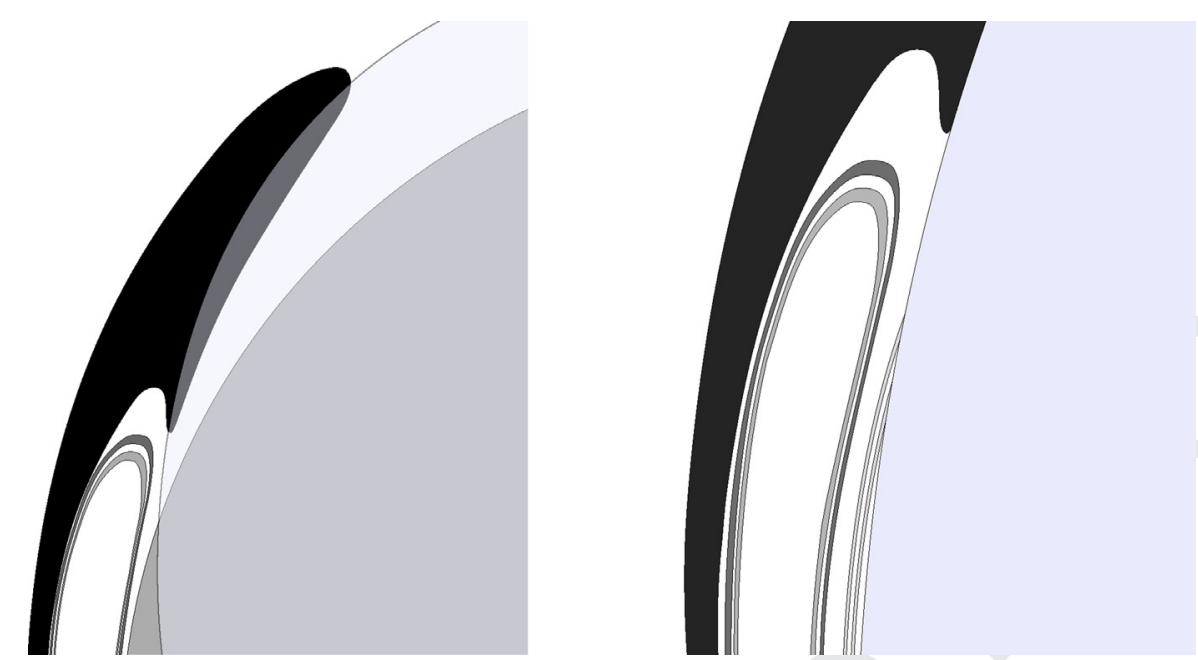

Fig. 7. Detail of the third section $(y=0, \dot{y}>0)$ of the stable manifold to the first quadrant periodic orbit for $h=0.024$. The section is composed of two simple tongues plus two complex ones. Here, we show one of the two simple tongues (in black) and one complex tongue (colored in tones of grey), a "Russian dollk" composed of an infinity of subtongues embedded inside each other. These subtongues are colored in tones of grey. We also represent in light blue the unstable manifold to the third quadrant periodic orbit. In the right panel, we reproduce a part of the infinite set of tongues infinitely winding around the stable manifold to the first quadrant periodic orbit. (For interpretation of the references to color in this figure legend, the reader is referred to the web version of this article.)
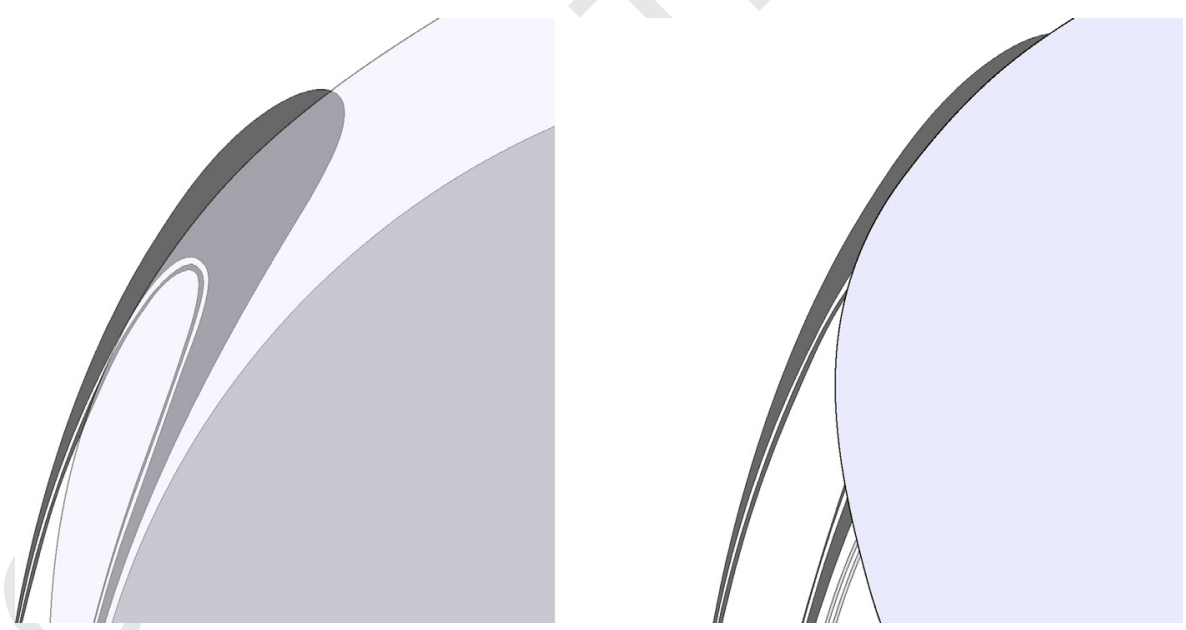

Fig. 8. Detail of the third section $(y=0, \dot{y}>0)$ of the stable manifold to the first quadrant periodic orbit for $h=0.0249$. The section is composed of two complex tongues. We show one of the two complex tongues (in grey), a "Russian doll" composed of an infinity of subtongues embedded inside each other. These subtongues are colored in grey. The orbits starting from the area shown in grey tones are mapped on the pieces of the tongues of the second section which are not inside the light blue area (Fig. 4, right panel, second row). We represent in light blue the unstable manifold to the third quadrant periodic orbit. In the right panel, we also reproduce a part of the infinite set of tongues infinitely winding around the stable manifold to the first quadrant periodic orbit (not represented here for the sake of simplicity). (For interpretation of the references to color in this figure legend, the reader is referred to the web version of this article.)

surface of section. The result of this intersection is shown in Fig. 9, for $h=0.024$ and $h=0.0249$. As we see, the scheme is the same as before. We have also represented (in light blue grey) the section of the unstable manifold to the upper-right periodic orbit. We observe that this manifold intersects the fourth section, but we will not analyze it as the procedure is basically the same than in the previous intersections.

In Figs. 10 and 11, we show the relation between the tongues in the third and fourth sections of the stable manifold to the first quadrant periodic orbit.

\section{Conclusions}

In this paper, we study the properties of the escape of orbits in a simple Hamiltonian model describing the motion near the centen of an elliptical galaxy. For certain values of the energy, the potential well opens up to infinity and test particles may escape. Every opening is bridged by an unstable periodic orbit called a Lyapunov orbit, which governs the escape 

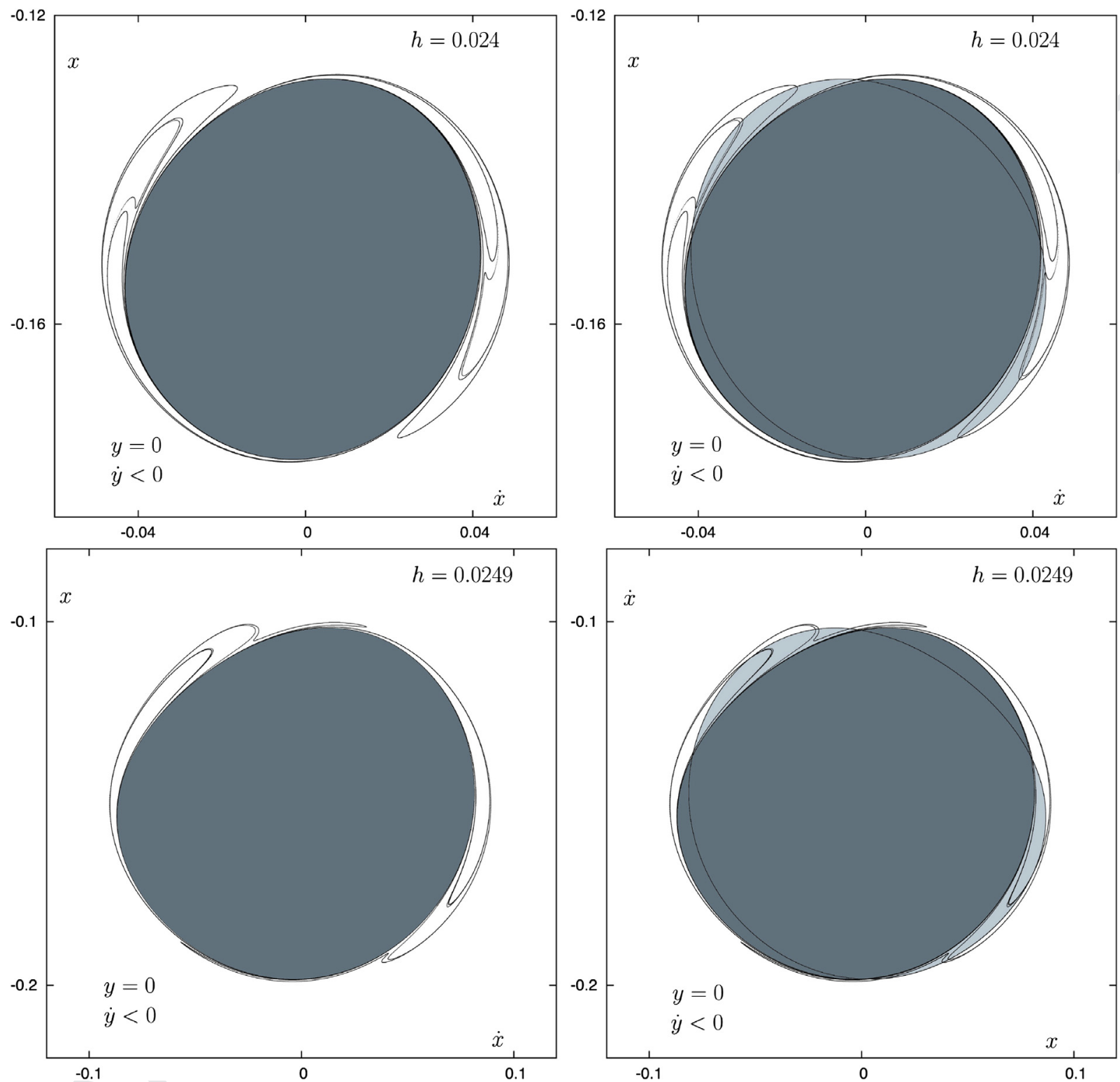

Fig. 9. Fourth section $(y=0, \dot{y}<0)$ of the stable manifold to the first quadrant periodic orbit, for $h=0.024$ (first row) and $h=0.0249$ (second row). As in Fig. 6, the section is composed by complex tongues composed of an infinity of subtongues embedded inside each other. All these tongues wind around the stable manifold to the third quadrant periodic orbit.

to infinity from the potential well. The sets of escaping orbits are limited by the stable and unstable manifolds to these periodic orbits. In [18], Navarro and Henrard computed the intersections of these manifolds with a surface of section in order to clarify the shapes and sizes of the windows of escape of stars. We found that in the system given by Hamiltonian (1), these windows consist of a "main windown" and of a hierarchy of secondary windows. A very large part of the main window is actually made of "just passing through" stars and may not be very interesting for galactic studies. Hence the importance of the secondary windows, their intricate spiral structures and the fractality of the basin boundaries.

Here, we have analyzed the same problem in a more complex Hamiltonian, given by (3). We have also investigated the intersections of the stable and unstable manifolds to the guardian periodic orbits with a surface of section, just to find the same mechanism governing the escape from the potential well. As in Hamiltonian (1), we have shown that the intricate spiral structures of the secondary windows governs the rate of escape: there are "first-ordex", infinitely winding spirals, but also "second ordex" spirals composed themselves of an infinity of layers, "third orde״" spirals formed by an infinity of second order spirals, and so on. 


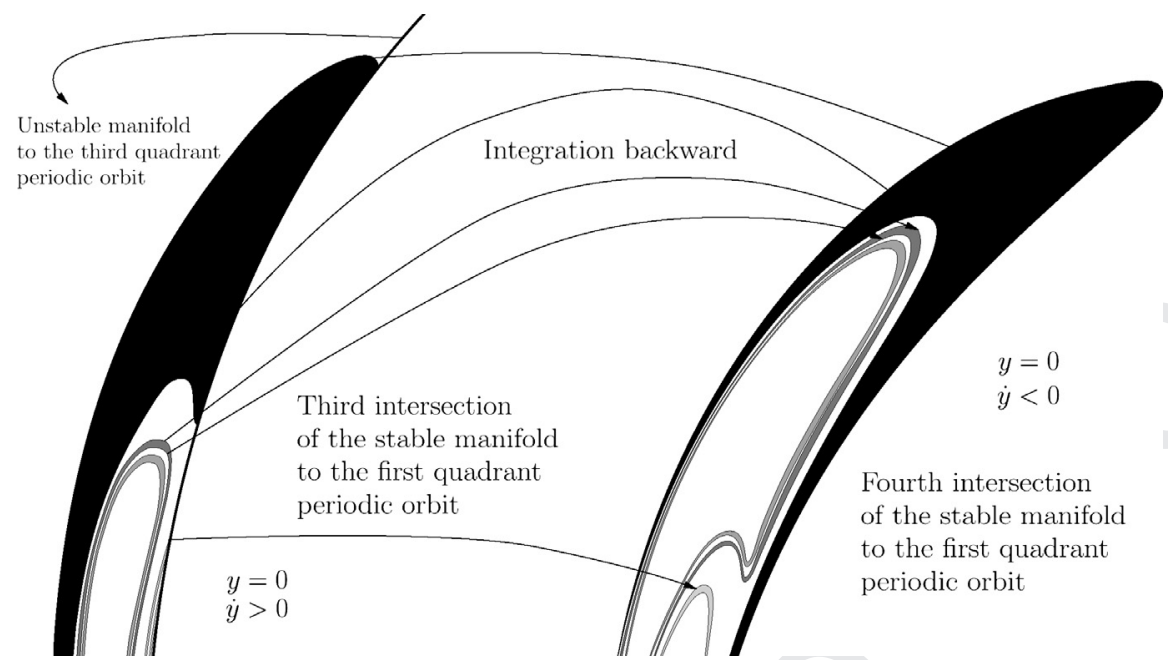

Fig. 10. Transformation of the tongues of the third section in the tongues of the fourth section integrating backward, for $h=0024$.

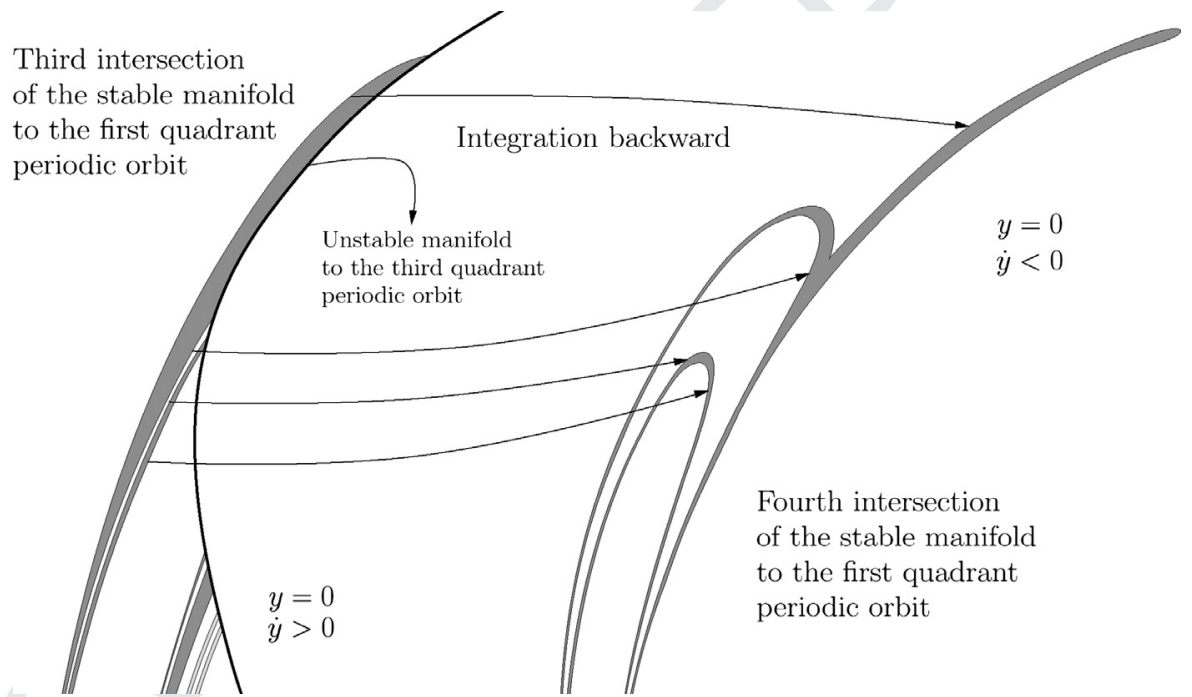

Fig. 11. Transformation of the tongues of the third section in the tongues of the fourth section integrating backward, for $h=00249$.

We have found that the number of "main" tongues in the third intersection of the stable manifold to the upper-right guardian periodic orbit with the surface of section $y=0, \dot{y}>0$ depends on the energy of the system. This is a consequence of the geometric shape of the tongues in the second section $(y=0, \dot{y}<0)$ of the stable manifold to the upper-right guardian periodic orbit (see Fig. 4, left panel), and the way this curve intersects with the unstable manifold to the upperright guardian periodic orbit. For $h=0.024$, the intersection originates four tongues, two simple and two complex. For $h=0.0249$, there are only two complex tongues, composed of subtongues embedded inside each other. In spite of the fact that these geometric differences between both values of the energy exist, we can conclude that the mechanism to explain the escape of stars from the potential well is the same that in the case of Hamiltonian (1).

\section{Uncited reference}

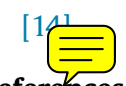

\section{References}

[1] J. Aguirre, J.C. Vallejo, M.A.F. Sanjuan, Wada basins and chaotic invariant sets in the Hénon-Heiles system, Phys. Rev. E 64 (2001) 11.066208.

[2] J. Aguirre, M.A.F. Sanjuan, Limit of small exits in open hamiltonian systems, Phys. Rev. E 67 (2003) 056201.

[3] J. Aguirre, R.L. Viana, M.A.F. Sanjuan, Fractal structures in nonlinear dynamics, Rev. Mod. Phys. 81 (2009) 333-386.

[4] B. Barbanis, Escape regions of a quartic potential, Celest. Mech. Dyn. Astron. 48 (1) (1990) 57-77.

[5] R. Barrio, F. Blesa, S. Serrano, Fractal structures in the Hénon-Heiles hamiltonian, Europhys. Lett. 82 (2008) 10003. 
209 [6] R. Barrio, F. Blesa, S. Serrano, Bifurcations and safe regions in open hamiltonians, New J. Phys. 11 (2009) 053004.

210 [7] R. Barrio, F. Blesa, S. Serrano, Bifurcations and chaos in hamiltonian systems, Int. J. Bifurcat. Chaos 20 (5) (2010) 1293-1319.

[8] F. Blesa, J.M. Seoane, R. Barrio, M.A.F. Sanjuan, To escape or not to escape, that is the question - perturbing the Hénon-Heiles hamiltonian, Int. J. Bifurcat. Chaos 22 (6) (2012) 1230010.

[9] N.D. Caranicolas, H. Varvoglis, Families of periodic orbits in a quartic potential, Astron. Astrophys. 141 (1984) $383-388$.

[10] N.D. Caranicolas, C.L. Vozikis, Order and chaos in galactic maps, Astron. Astrophys. 349 (1999) 70.

[11] G. Contopoulos, Asymptotic curves and escapes in hamiltonian systems, Astron. Astrophys. 231 (1) (1990) $41-45$.

[12] G. Contopoulos, D. Kaufmann, Types of escapes in a simple hamiltonian system, Astron. Astrophys. 253 (2) (1992) $379-388$.

[13] G. Contopoulos, K. Efstathiou, Escapes and recurrence in a simple hamiltonian system, Celest. Mech. Dyn. Astron. 88 (2) (2004) 163-183.

A. Deprit, J. Henrard, A manifold of periodic orbits, Adv. Astron. Astrophys. 6 (1968) 1-124.

A. Deprit, J. Henrard, Construction of orbits asymptotic to a periodic orbit, Astron. J. 74 (1969) 308-316.

[16] A. Deprit, J.F. Price, Numerical integration by recurrent power series, Astron. Astrophys. 1 (1969) 427.

[17] A.P.S. de Moura, P.S. Letelier, Fractal basins in Henon-Heiles and other polynomial potentials, Phys. Lett. A 256 (1999) $362-368$.

[18] J.F. Navarro, J. Henrard, Spiral windows for escaping stars, Astron. Astrophys. 369 (2001) 1112-1121.

[19] C. Siopsis, H.E. Kandrup, G. Contopoulos, R. Dvorak, Universal properties of escape in dynamical systems, Celest. Mech. Dyn. Astron. 65 (1-2) (1996) 57-68.

[20] F. Verhulst, Discrete symmetric dynamical systems at the main resonances with applications to axi-symmetric galaxies, Phil. Trans. R. Soc. Lond. 290 (1375) (1979) 435-465.

[21] E.E. Zotos, Trapped and escaping orbits in an axially symmetric galactic-type potential, PASA 29 (2012) 161-173.

[22] E.E. Zotos, Escape dynamics in a hamiltonian system with four exit channels, Nonlinear Stud. 22 (3) (2015) 1-20. 